\title{
Phase I trial of oxaliplatin plus S-1 chemotherapy in patients with metastatic colorectal cancer
}

\author{
MANABU EMI, YOSHIYUKI YAMAGUCHI, JUN HIHARA, KATSUJI HIRONAKA and MORIHITO OKADA \\ Department of Surgical Oncology, Research Institute for Radiation Biology and Medicine, \\ Hiroshima University, Hiroshima 734-8553, Japan
}

Received August 4, 2009; Accepted October 12, 2009

DOI: $10.3892 /$ ol_00000017

\begin{abstract}
Advanced colorectal cancer can be effectively treated with $\mathrm{S}-1$, as well as with a combination of oxaliplatin, 5-fluorouracil (5FU) and leucovorin (LV). However, S-1 together with oxaliplatin may provide a more convenient alternative to $5 \mathrm{FU} / \mathrm{LV}$. To evaluate the performance of S-1 combined with oxaliplatin for patients with colorectal cancer, we conducted a Phase I clinical trial in an outpatient setting. We administered S-1 to 15 patients with advanced colorectal adenocarcinoma for two weeks followed by one week of rest. Oxaliplatin was also administered on day 1 of the $\mathrm{S}-1$ cycle. The dose of oxaliplatin was increased from 40 to $85 \mathrm{mg} / \mathrm{m}^{2}$ to define the maximum tolerated dose and recommended dose in preparation for a Phase II trial. We administered 102 courses of treatment to 15 patients. Grade 3 thrombocytopenia developed in only 1 patient at a dose of $85 \mathrm{mg} / \mathrm{m}^{2}$ of oxaliplatin plus oral S-1. No other grade 3-4 toxicities developed. No dose-limiting toxicity developed at level 4 of our regimen (oxaliplatin $85 \mathrm{mg} / \mathrm{m}^{2}$ ), and the recommended dose for a Phase II trial was $85 \mathrm{mg} / \mathrm{m}^{2}$ of oxaliplatin in an outpatient setting.
\end{abstract}

\section{Introduction}

The mainstay of therapy for patients with metastatic colorectal cancer is 5-fluorouracil (5FU), which confers a survival benefit over the best supportive care (1).

Although traditionally administered as a single agent, the effects of 5FU progressively improved through combination with leucovorin (LV) (2), as well as oxaliplatin $(3,4)$.

In particular, recently published results from the Intergroup N9741 study comparing the de Gramont oxaliplatin/5-FU/ LV (original Saltz IFL) regimen suggest a survival benefit and superior time to progression and response rates with

Correspondence to: Dr Manabu Emi, Department of Surgical Oncology, Research Institute for Radiation Biology and Medicine, Hiroshima University, Kasumi 1-2-3, Minami-Ku, Hiroshima 734-8553, Japan

E-mail: moaista@wish.ocn.ne.jp

Key words: metastatic colorectal cancer, S-1, oxaliplatin oxaliplatin-based therapy compared with irinotecan-based strategies (5). However, the schedule for delivering these regimens is cumbersome and requires central venous access.

S-1 is a novel orally administered drug comprising tegafur, 5-chloro-2, 4-dihydroxypyridine (CDHP) and oteracil potassium (Oxo) at a 1:0.4:1 molar concentration ratio (6). Its antitumor effect is achieved by the 5FU prodrug tegafur, while CDHP competitively inhibits the 5FU degradative enzyme dihydropyrimidine dehydrogenase (DPD), resulting in the prolonged circulation of a higher blood 5FU concentration (7). Oxo competitively inhibits the orotate phosphorylation of $5 \mathrm{FU}$ in the gastrointestinal tract and thus reduces the serious gastrointestinal toxicity associated with 5FU (8).

Several clinical trials of S-1 monotherapy showed the doselimiting toxicities of myelosuppression in Japanese studies and diarrhea in European and North American studies (9-11). Phase II trials of S-1 as a single agent have revealed response rates ranging from 19 to $39 \%$ among patients with advanced colorectal cancer (12-14). These findings demonstrated that the response rates to $\mathrm{S}-1$ are high and that compliance is good among patients with advanced colorectal cancer treated on an outpatient basis.

S-1 should be tested in combination with oxaliplatin considering its documented effects and more convenient administration compared with 5FU/LV.

We therefore conducted a Phase I/II clinical study of S-1 combined with oxaliplatin. Our primary objectives were to estimate the maximum tolerated dose (MTD) of the combination and to determine the recommended dose (RD) for Phase II studies. The clinical activity and feasibility of this chemotherapy regimen were investigated in the Phase II study.

\section{Patients and methods}

Criteria. The criteria for participation included histologically or cytologically confirmed advanced or recurrent colorectal adenocarcinoma with measurable metastatic lesions. Other criteria included age between 20 and 75 years, Eastern Cooperative Group (ECOG) performance status 0-2, adequate baseline bone marrow (white blood cell count between 4,000 and $10,000 / \mu \mathrm{l}$ and platelets $\geq 100,000 / \mu \mathrm{l}$ ), suitable hepatic function [bilirubin serum level $<1.25 \mathrm{x}$ the upper normal limit (UNL) and serum aminotransferases $<2.5 \times \mathrm{UNL}$, and 
suitable renal function (serum creatinine level $<1.5 \times$ UNL). Exclusion criteria included diarrhea, uncontrolled infection, symptomatic brain metastasis, bowel obstruction or a high risk of a poor outcome due to concomitant uncontrollable non-malignant diseases such as diabetes, cardiac failure or renal failure. Patients who had undergone prior oxaliplatin therapy were also excluded. The institutional review board of Hiroshima University Hospital approved the study (no. 451), and all eligible patients provided written informed consent before enrollment.

Treatment schedule. The fixed dose of S-1 was $80 \mathrm{mg} / \mathrm{m}^{2} /$ day. Three S-1 doses were established according to body surface area (BSA) as follows: BSA $<1.25 \mathrm{~m}^{2}, 80 \mathrm{mg} /$ day; BSA $1.25-1.5 \mathrm{~m}^{2}, 100 \mathrm{mg} /$ day and BSA $\geq 1.5 \mathrm{~m}^{2}, 120 \mathrm{mg} /$ day. Patients received half of their assigned dose after breakfast and the other half after dinner orally (p.o.). Oxaliplatin was administered as an intravenous (i.v.) infusion in $500 \mathrm{ml}$ of $5 \%$ dextrose over a period of $2 \mathrm{~h}$ on day 1 . The oxaliplatin dose was escalated in increments of $15 \mathrm{mg} / \mathrm{m}^{2}$ as follows: 40, 55, 70 and $85 \mathrm{mg} / \mathrm{m}^{2}$ for each cohort. Intra-patient dose escalation was not permitted. To prevent nausea and vomiting, routine administration of a standard dose of a 5-HT3 antagonist occurred at the time of oxaliplatin administration. The protocol was repeated until tumor progression or dose-limiting toxicity (DLT) occurred as described below, or the patient refused further treatment.

We defined DLT as grade 3 or 4 non-hematological toxicities excluding nausea, vomiting, hyperglycemia and sodium, potassium and calcium abnormalities; grade 3 neutropenia and thrombocytopenia during the first course. Each dose level comprised a minimum of 3 patients. If the 3 patients at level 1 completed one cycle of treatment without DLT, the following 3 patients were entered at level 2 . If 1 of the 3 patients developed DLT, 3 additional patients were recruited at the same dose level. If 2 of 3, or 2 of 6 patients had DLT, the MTD was defined as the dose given to this cohort. The RD was defined as one level below MTD. If neither level met the criteria for MTD, the RD of oxaliplatin was defined as $85 \mathrm{mg} / \mathrm{m}^{2}$.

Toxicity and response evaluation. The primary endpoint was DLT, and the secondary endpoint was to evaluate potential antitumor activity.

Patient evaluation. We assessed the history of disease and the general condition of each patient based on interviews, physical examinations and blood tests before enrollment in the study. Toxicity was assessed according to the National Cancer Institute Common Toxicity Criteria (NCI-CTC), version 3.0. During treatment, toxicity was assessed weekly during the first and subsequent cycles. The responses of assessable disease sites were evaluated in patients who received at least two cycles of treatment according to RECIST criteria (15).

\section{Results}

Patient characteristics. Table I lists the characteristics of the 15 patients who entered this study between September 2005 and February 2007. The median age of the patients was 56 years, and all but 2 of them had an ECOG performance
Table I. Patient characteristics.

\begin{tabular}{lc}
\hline No. of patients & 15 \\
Colon/rectum & $6 / 9$ \\
Male/female & $13 / 2$ \\
Age (years) & 56 \\
Median & $41-79$ \\
Range & $13 / 2$ \\
Performance status (ECOG) 0/1 & \\
Metastatic site & 3 \\
Primary site & 7 \\
Liver & 1 \\
Lung & 3 \\
Abdominal lymph nodes & 2 \\
Peritoneum & \\
Previous therapy & 9 \\
Surgical resection & 4 \\
5FU/LV chemotherapy & 5 \\
CPT-11 chemotherapy & 3 \\
None &
\end{tabular}

ECOG, Eastern Cooperative Group; 5FU, 5-fluorouracil; LV, leucovorin.

status of 0 . Nine patients had undergone prior resection of their primary tumors. Four had undergone adjuvant LV plus a bolus 5FU injection (the RPMI regimen) and 5 had undergone first-line chemotherapy using CPT-11 (for example, FOLFIRI). Patients were eligible for toxicity evaluations at any time during delivery of the 102 courses (median 6 courses; range 2-11).

Toxicity. Table II lists the toxicities observed in the 15 enrolled patients. With regard to overall hematological toxicity, grade 3 thrombocytopenia developed in only 1 patient at level 4 , and other grade 3-4 toxicities were not evident. No grade 3-4 non-hematological toxicities developed. The most frequent non-hematological toxicity was neuropathy, which affected $67 \%$ of the patients at level 4 .

No DLT developed at a dose level of 1-3. One patient developed grade 3 thrombocytopenia at a dose level of 4 , but the other 2 patients in the same cohort did not develop DLT. An additional 3 patients were enrolled for a safety evaluation, but these 3 patients did not develop DLT. Therefore, level 4 ( $85 \mathrm{mg} / \mathrm{m}^{2}$ of oxaliplatin) was confirmed as the RD for our regimen.

Effects. Patients were included in the response evaluation. Of the 10 patients (first-line chemotherapy group), 5 responded [2 complete response (CR) and 3 partial response (PR)] to yield an overall response rate of $50 \%$. Table III shows the response according to the oxaliplatin dose. Three patients had stable disease (SD) and $90 \%$ of the patients in this cohort derived a clinical benefit $(\mathrm{CR}+\mathrm{PR}+\mathrm{SD})$. The median time to progression was 214 days. Among 5 patients who had undergone first-line chemotherapy using CPT-11 (second-line 
Table II. Toxicity.

Oxaliplatin dose

\begin{tabular}{|c|c|c|c|c|c|c|c|}
\hline \multicolumn{2}{|c|}{$40 \mathrm{mg} / \mathrm{m}^{2}(\mathrm{n}=3)$} & \multicolumn{2}{|c|}{$55 \mathrm{mg} / \mathrm{m}^{2}(\mathrm{n}=3)$} & \multicolumn{2}{|c|}{$70 \mathrm{mg} / \mathrm{m}^{2}(\mathrm{n}=3)$} & \multicolumn{2}{|c|}{$85 \mathrm{mg} / \mathrm{m}^{2}(\mathrm{n}=6)$} \\
\hline $\mathrm{Gl} / 2$ & G3 & $\mathrm{Gl} / 2$ & G3 & $\mathrm{Gl} / 2$ & G3 & $\mathrm{Gl} / 2$ & G3 \\
\hline
\end{tabular}

Hematological

Anorexia

Nausea/vomiting

Hand-foot syndrome

Diarrhea

Rash

Alopecia

Neuropathy

Hepatobilliary

1
1
0
0
1
1
0
0
0

0

$0 \quad 2$

10

0

0

$\begin{array}{lll}0 & 1 & 0\end{array}$

$0 \quad 0$

0

$0 \quad 0$

$0 \quad 0$

0

Non-hematological

\begin{tabular}{|c|c|c|c|c|c|c|c|c|}
\hline Leukopenia & 0 & 0 & 0 & 0 & 0 & 0 & 2 & 0 \\
\hline Neutropenia & 0 & 0 & 0 & 0 & 0 & 0 & 2 & 0 \\
\hline Thrombocytopenia & 2 & 0 & 2 & 0 & 1 & 0 & 3 & 1 \\
\hline Anemia & 0 & 0 & 0 & 0 & 0 & 0 & 1 & 0 \\
\hline
\end{tabular}

Table III. Tumor responses.

\begin{tabular}{|c|c|c|c|c|}
\hline & \multicolumn{4}{|c|}{ Oxaliplatin dose } \\
\hline & $40 \mathrm{mg} / \mathrm{m}^{2}(\mathrm{n}=3)$ & $55 \mathrm{mg} / \mathrm{m}^{2}(\mathrm{n}=3)$ & $70 \mathrm{mg} / \mathrm{m}^{2}(\mathrm{n}=3)$ & $85 \mathrm{mg} / \mathrm{m}^{2}(\mathrm{n}=6)$ \\
\hline \multicolumn{5}{|l|}{ First-line } \\
\hline Complete response & 1 & 1 & - & - \\
\hline Partial response & - & - & 1 & 2 \\
\hline Stable disease & 2 & - & 1 & - \\
\hline Progressive disease & - & - & - & 1 \\
\hline \multicolumn{5}{|c|}{ Objective response rate $50 \%$; median time to progression 214 days } \\
\hline \multicolumn{5}{|l|}{ Second-line } \\
\hline Complete response & - & - & - & - \\
\hline Partial response & - & - & - & 1 \\
\hline Stable disease & - & - & - & 1 \\
\hline Progressive disease & - & 2 & 1 & 1 \\
\hline
\end{tabular}

chemotherapy group), 1 achieved a partial response. The median time to progression was 72 days.

\section{Discussion}

We conducted a Phase I trial of oxaliplatin combined with oral S-1 chemotherapy for metastatic colorectal cancer in an outpatient setting. Non-hematological toxicities including fatigue, anorexia and neuropathy were well-tolerated and controlled, indicating that this treatment is feasible in the outpatient setting. Grade 3 thrombocytopenia occurred at 85 $\mathrm{mg} / \mathrm{m}^{2}$ of oxaliplatin plus oral $\mathrm{S}-1$ but none of the remaining 5 patients in the same cohort developed DLT. MTD was not achieved. Thus, RD of oxaliplatin was defined as $85 \mathrm{mg} / \mathrm{m}^{2}$, since we aimed to develop a long-term tolerable regimen. Indeed, all cases were continued safely until the development of progressive disease, and CR was achieved at the dose of level 1 and 2.

Other investigators have examined regimens that combined oral fluoropyrimidine, capecitabine and oxaliplatin. Cassidy et al (16) reported the results of a Phase III study of capecitabine plus oxaliplatin (XELOX) compared with 5-FU/LV plus 
oxaliplatin (FOLFOX-4) as first-line therapy for metastatic colorectal cancer. Oxaliplatin $\left(130 \mathrm{mg} / \mathrm{m}^{2}\right)$ was administered on day 1 and capecitabine $\left(2,000 \mathrm{mg} / \mathrm{m}^{2}\right)$ was delivered for 14 days with a 1 -week rest, every 3 weeks. The effect of XELOX was statistically similar to that of FOLFOX-4: median progression-free survival, 8.0 vs. 8.5 months and median overall survival, 19.8 vs. 19.6 months. Grade 3 or 4 diarrhea appeared in $20 \%$ of patients administered with XELOX, and in $11 \%$ of those administered with FOLFOX4. Grade 3 handfoot syndrome (HFS) appeared in $6 \%$ of those administered with XELOX and in $1 \%$ of those given FOLFOX4. Grade 3 or 4 neutropenia developed in 6 and $43 \%$ of patients admistered with XELOX and FOLFOX4, respectively. The Phase III study by Rothenberg et al (17) showed that XELOX was statistically as effective as FOLFOX-4 when administered as a second-line treatment in patients with metastatic colorectal cancer (median progression-free survival, 4.7 vs. 4.8 months; median overall survival, 11.9 vs. 12.5 months). In summary, the results of these Phase III studies showed that capecitabine could replace 5FU/LV infusions.

Fewer doses of oxaliplatin were delivered in our regimen compared with the XELOX regimen. However, we achieved a complete response in each patient at levels 1 and 2 (40 and $55 \mathrm{mg} / \mathrm{m}^{2}$, respectively) of oxaliplatin. Grade $3 \mathrm{HFS}$ developed in $6 \%$ of patients on XELOX but not in any patients at the RD of our oxaliplatin plus S-1 (SOX) regimen. The latter may be due to the low level of $\beta$-alanine induced by the DPD inhibitory property of S-1 (18). Since the severity of thrombocytopenia depends on the dose of oxaliplatin, the incidence of thrombocytopenia appears to be higher in the reported SOX regimen with oxaliplatin at a dose of $130 \mathrm{mg} / \mathrm{m}^{2}$ (19). Nevertheless, our SOX regimen continued without prolonged thrombocytopenia. These results indicated that our SOX regimen is highly feasible and comparable to the XELOX regimen. Thus, our SOX regimen should be evaluated in the next Phase II trial, since compliance is a critical factor in the outcome of chemotherapy for patients with metastatic colorectal cancer.

In conclusion, we examined the effects of an escalating dose of oxaliplatin i.v. combined with S-1 p.o. for 14 days over a 3-week treatment cycle. No DLTs developed at a level 4 dose (oxaliplatin $85 \mathrm{mg} / \mathrm{m}^{2}$ ), and the RD for a Phase II trial was $85 \mathrm{mg} / \mathrm{m}^{2}$ of oxaliplatin in an outpatient setting. The benefits of mild chemotherapy in terms of tumor response, patient survival and quality of life are receiving increasing attention. Our treatment regimen may be a safe, effective, practical and, therefore, beneficial regimen for patients with incurable metastatic colorectal cancer.

\section{References}

1. Scheithauer W, Rosen H, Kornek GV, Sebesta C and Depisch D: Randomised comparison of combination chemotherapy plus supportive care with supportive care alone in patients with metastatic colorectal cancer. BMJ 306: 752-755, 1993.

2. Thirion P, Michiels S, Pignon JP, et al: Modulation of fluorouracil by leucovorin in patients with advanced colorectal cancer: an updated meta-analysis. J Clin Oncol 22: 3766-3776, 2004.
3. De Gramont A, Figer A, Seymour M, et al: Leucovorin and fluorouracil with or without oxaliplatin as first-line treatment in advanced colorectal cancer. J Clin Oncol 18: 2938-2947, 2000.

4. Giacchetti S, Perpoint B, Zidani R, et al: Phase III multicenter randomized trial of oxaliplatin added to chronomodulated fluorouracil-leucovorin as first-line treatment of metastatic colorectal cancer. J Clin Oncol 18: 136-147, 2000

5. Goldberg RM, Sargent DJ, Morton RF, et al: Randomized controlled trial of reduced-dose bolus fluorouracil plus leucovorin and irinotecan or infused fluorouracil plus leucovorin and oxaliplatin in patients with previously untreated metastatic colorectal cancer: a North American Intergroup Trial. J Clin Oncol 24: 3347-3353, 2006.

6. Shirasaka T, Nakano K, Takechi T, et al: Antitumor activity of $1 \mathrm{M}$ tegafur-0.4 M 5-chloro-2,4-dihydroxypyridine-1 M potassium oxonate (S-1) against human colon carcinoma orthotopically implanted into nude rats. Cancer Res 56: 2602-2606, 1996.

7. Tatsumi K, Fukushima M, Shirasaka T and Fujii S: Inhibitory effects of pyrimidine, barbituric acid and pyridine derivatives on 5 -fluorouracil degradation in rat liver extracts. Jpn J Cancer Res 78: 748-755, 1987.

8. Shirasaka T, Shimamato Y, Ohshimo H, Yamaguchi M, Kato T, Yonekura K and Fukushima M: Development of a novel form of an oral 5-fluorouracil derivative (S-1) directed to the potentiation of the tumor selective cytotoxicity of 5-fluorouracil by two biochemical modulators. Anticancer Drugs 7: 548-557, 1996.

9. Sugimachi K, Maehara Y, Horikoshi N, Shimada Y, Sakata Y, Mitachi Y and Taguchi T: An early phase II study of oral S-1, a newly developed 5-fluorouracil derivative for advanced and recurrent gastrointestinal cancers. The S-1 Gastrointestinal Cancer Study Group. Oncology 57: 202-210, 1999.

10. Hoff PM, Saad ED, Ajani JA, et al: Phase I study with pharmacokinetics of S-1 on an oral daily schedule for 28 days in patients with solid tumors. Clin Cancer Res 9: 134-142, 2003.

11. Van Groeningen CJ, Peters GJ, Schornagel JH, et al: Phase I clinical and pharmacokinetic study of oral S-1 in patients with advanced solid tumors. J Clin Oncol 18: 2772-2779, 2000.

12. Ohtsu A, Baba H, Sakata Y, Mitachi Y, Horikoshi N, Sugimachi K and Taguchi T: Phase II study of S-1, a novel oral fluoropyrimidine derivative, in patients with metastatic colorectal carcinoma. S-1 Cooperative Colorectal Carcinoma Study Group. Br J Cancer 83: 141-145, 2000.

13. Shirao K, Ohtsu A, Takada H, et al: Phase II study of oral S-1 for treatment of metastatic colorectal carcinoma. Cancer 100: 2355-2361, 2004.

14. Van den Brande J, Schöffski P, Schellens JH, et al: EORTC Early Clinical Studies Group early phase II trial of S-1 in patients with advanced or metastatic colorectal cancer. Br J Cancer 88: 648-656, 2003.

15. Therasse P, Arbuck SG, Eisenhauer EA, et al: New guidelines to evaluate the response to treatment in solid tumors. European Organization for Research and Treatment of Cancer, National Cancer Institute of the United States, National Cancer Institute of Canada. J Natl Cancer Inst 92: 205-216, 2000.

16. Cassidy J, Clarke S, Díaz-Rubio E, et al: Randomized phase III study of capecitabine plus oxaliplatin compared with fluorouracil/ folinic acid plus oxaliplatin as first-line therapy for metastatic colorectal cancer. Clin Oncol 26: 2006-2012, 2008.

17. Rothenberg ML, Cox JV, Butts C, et al: Capecitabine plus oxaliplatin (XELOX) versus 5-fluorouracil/folinic acid plus oxaliplatin (FOLFOX-4) as second-line therapy in metastatic colorectal cancer: a randomized phase III noninferiority study. Ann Oncol 19: 1720-1726, 2008.

18. Yamada Y, Hamaguchi T, Goto M, et al: Plasma concentrations of 5-fluorouracil and F-alanine following oral administration of S-1, a dihydropyrimidine dehydrogenase inhibitory fluoropyrimidine, as compared with protracted venous infusion of 5-fluorouracil. Br J Cancer 89: 816-820, 2003.

19. Yamada Y, Tahara M, Miya T, et al: Phase I/II study of oxaliplatin with oral S-1 as first-line therapy for patients with metastatic colorectal cancer. Br J Cancer 98: 1034-1038, 2008. 